\title{
Spin-twist driven persistent current in a strongly correlated two-dimensional electron system: \\ a manifestation of the gauge field
}

\author{
K. Kusakabe \\ Institute for Solid State Physics, University of Tokyo, Roppongi, Tokyo 106, Japan \\ H. Aoki \\ Department of Physics, University of Tokyo, Hongo, Tokyo 113, Japan
}

\begin{abstract}
A persistent current, coupled with the spin state, of purely manybody origin is shown to exist in Nagaoka's ferromagnetic state in two dimensions (2D). This we regard as a manifestation of a gauge field, which comes from the surrounding spin configuration and acts on the hole motion, being coupled to the Aharonov-Bohm flux. This provides an example where the electron-electron interaction exerts a profound effect involving the spins in clean two-dimensional lattice systems in sharp contrast to continuum or spinless fermion systems.

PACS numbers: 72.10.Bg, 05.30.Fk, 73.20.Dx
\end{abstract}

Typeset using REVTEX 
Persistent current is a highlight of the physics of electrons on mesoscopic scales. One major interest is how the electron-electron interaction affects the persistent current. The issue was originally posed to answer whether the interaction can offset the reduction of the current due to disorder. The many-body effect has been examined for both continuum systems and lattice systems with various geometries such as a one-dimensional ring or a torus $\| 1-9]$.

In clean continuum systems, however, the electron-electron interaction, by itself, exerts null effect on the persistent current, which is exactly shown in one dimension (1D) and expected to persist in two dimensions (2D) and higher ones, unless the interaction creates low-energy excitations below the lowest excitation of the noninteracting system [5].

The lattice system is a different story, even when clean, for two reasons. First, a lattice system can undergo Mott's metal-insulator transition at some band filling or at some critical interaction strength, so that the interaction may suppress the persistent current around the transition [10,4].

However, a truly interesting effect of interaction in lattice systems should appear when one considers the spin degrees of freedom. An effect of spin is known to appear as a change in the Aharonov-Bohm (AB) period (in units of the flux quantum, $\Phi_{0}=h c / e$ ) and amplitude, originally shown for the noninteracting model by averaging over the number of electrons [11] and later found in interacting systems such as the one-dimensional Hubbard model with a fixed number of electrons in the dilute and/or strong-coupling limit. [12 Change in the orbital magnetism and current is also found in the finite- $U$ Hubbard model with the spin-orbit interaction [13].

In the present paper we show that an interplay between charge and spin degrees of freedom can result in a qualitatively different phenomenon, i.e., a persistent current "driven by" a twist in the spin structure with the external flux in a strong- $U$ Hubbard model in $2 \mathrm{D}$.

In strongly correlated systems, the ground state is often sensitive to the boundary condition. For instance, Nagaoka's ferromagnetic ground state [14], which is an extreme manifestation of the correlation for large $U$ in the immediate (one-hole doped) vicinity of the 
Mott insulator, evolves into a spin-singlet state when the antiperiodic boundary condition is adopted in one direction. [15] We can then expect that the boundary-condition dependence will signify a large response to the $\mathrm{AB}$ flux and a persistent current via the Byers-Yang theorem. [16] This does indeed turn out to be the case. We shall trace this back to a gauge potential inherent in lattice systems, where the gauge potential has an ability to cope with the frustration caused by the twisted boundary condition, a situation distinct from continuum systems.

We consider the two-dimensional Hubbard model on a torus to get rid of edges, and pierce an $\mathrm{AB}$ flux $\Phi$ through the opening. This amounts to a frustration, or a change in the boundary condition along $y$ for the wave function $\phi$ to

$$
\phi\left(\ldots, \mathbf{x}_{i}+N_{y} \mathbf{u}_{y}, \ldots\right)=\exp \left(\mathrm{i} 2 \pi \Phi / \Phi_{0}\right) \phi\left(\ldots, \mathbf{x}_{i}, \ldots\right)
$$

where $\mathbf{x}_{i}$ is the position of the $i$ th electron, and $\mathbf{u}_{a}\left(N_{a}\right)$ the unit vector (the number of sites) in the $a(=x, y)$ direction with the lattice constant being unity.

We have numerically obtained the change in the energy spectrum with $\Phi$ at $U=\infty$ for 15 (14) electrons on a $4 \times 4$-site system, i.e., one (two) -hole doped from half filling) in Fig. 1. We immediately recognize that there is a dramatic series of level crossings for low-lying states in such a way that the total spin of the ground state $S$ oscillates between Nagaoka's full polarization (which occurs at $\Phi=0$ for one hole or at the half-flux for two holes) and vanishing $(S \simeq 0)$ polarization.

This oscillation gives rise to a persistent current I (Fig. 2) via the Byers-Yang theorem, 16

$$
I=-c \frac{\partial\langle\phi|H(\Phi)| \phi\rangle}{\partial \Phi}
$$

where $|\phi\rangle$ is assumed to be normalized. Thus, not only the magnitude but the overall profile of the current deviate from the spinless electron system (the curve for the fully polarized state).

The flux-induced oscillation in $S$ found here is totally incompatible with the ordinary Stoner ferromagnetism. In the Stoner picture, the lowest charge excitation with a spin flip is 
the Stoner excitation, for which a Stoner gap opens over the whole Brillouin zone for a fully polarized state, so that the Stoner excitation cannot contribute to the persistent current. The collective spin wave also remains in an excited state when $\Phi$ is varied. For partially polarized states, the Stoner excitation continuum becomes gapless and a finite $\Phi$ can induce single spin flips, but this will only result in a change of $S$ by unity. In Nagaoka's state, by contrast, $S$ oscillates between the largest and smallest possible values, which is due to an anomaly in the gap and spin stiffness where the spin mass vanishes like the inverse system size 14,17,18.

In a doped Mott insulator, which we have at hand, a fundamental deviation from the spinless fermions arises from an interplay of the charge (hole) motion and the surrounding spin configuration, which is indeed responsible for anomalous excitations. To show this we can exploit the formulation of Ref. [20], where we write down an eigenfunction of the one-hole system in a "Bloch's form" as

$$
|\phi\rangle=\sum_{\mathbf{x}_{\mathrm{h}}} \exp \left(\mathrm{ik} \cdot \mathbf{x}_{\mathrm{h}}\right) \sum_{\left\{\sigma_{j}\right\}} f\left(\sigma_{1}, \ldots, \sigma_{N_{\mathrm{e}}}\right) c_{\mathbf{x}_{1}, \sigma_{1}}^{\dagger} \cdots c_{\mathbf{x}_{N_{\mathrm{e}}}, \sigma_{N_{\mathrm{e}}}}^{\dagger}|0\rangle .
$$

Here $\mathbf{x}_{\mathrm{h}}$ is the position of the hole, $c_{\mathbf{x}_{j}, \sigma_{j}}^{\dagger}$ creates an electron with spin $\sigma_{j}$ at $\mathbf{x}_{j}$, and $N_{\mathrm{e}}$ $(=N-1)$ is the number of electrons in an $N$-site system. The advantage of working with a spin wavefunction $f\left(\sigma_{1}, \ldots, \sigma_{N_{\mathrm{e}}}\right)$ around the hole is the following. If the order $\left(\mathbf{x}_{1}, \ldots, \mathbf{x}_{N_{\mathrm{e}}}\right)$ according to which the creation operators are multiplied, is specified by the coordinate relative to $\mathbf{x}_{\mathrm{h}}, f$ no longer contains $\mathbf{x}_{\mathrm{h}}$, and the equation determining the spin configuration around the hole is simplified: A hole hopping from $\mathbf{x}_{\mathrm{h}}$ to $\mathbf{x}_{\mathrm{h}}+\mathbf{u}_{a}$ is mapped onto a spin operation that is almost a uniform translation of the spins in the inverse direction, except for the spin at $\mathbf{x}_{\mathrm{h}}+\mathbf{u}_{a}$, which has to move to $\mathbf{x}_{\mathrm{h}}-\mathbf{u}_{a}$. If we write the whole operation as $\mathcal{T}_{a}$, we end up with a spin Hamiltonian,

$$
\mathcal{H}_{\text {spin }}=-t \sum_{a=1}^{d}\left\{\exp \left(i \mathbf{k} \cdot \mathbf{u}_{a}\right) \mathcal{T}_{a}+\exp \left(-i \mathbf{k} \cdot \mathbf{u}_{a}\right) \mathcal{T}_{a}^{-1}\right\}
$$

In this formalism, $\Phi$ simply shifts the $k$ points uniformly as $\mathbf{k} \rightarrow \mathbf{k}+\left(0,2 \pi \Phi /\left(N_{y} \Phi_{0}\right)\right)$.

We can then identify the origin of the many-body effect on the current as the gauge 
potential in the following sense. When we decompose the motion of electrons into the centerof-mass and internal ones, $\Phi$ only couples to the former, which is completely decoupled from the latter for continuum systems. Thus, a persistent current cannot deviate from the freefermion behavior [5]. Even if the interaction creates low-energy excitations leading to level crossings for finite $\Phi$, the curvature of the spectral flow $\left[E_{i}(\Phi)\right]$ does not deviate from that for the free fermions.

In sharp contrast, the center-of-mass motion does disturb the relative coordinates on a lattice. Namely, a shift in $\mathbf{x}_{\mathrm{h}}$, which coincides with the center-of-mass here, affects the relative coordinates between electrons through the electron correlation (i.e., excluded double occupancies). In the present formalism, the coupling of the two coordinates appears as a discrepancy of $\mathcal{T}_{a}$ from the uniform translation. [19] The spin wave function is thus affected by the flux-coupled charge motion, and changes its structure with $\Phi$ in two- (or higher) dimensional lattice systems.

Here we notice that the spin configuration does not flow into an antiferromagnetic (AF) state with $\Phi$, since this would impede the motion of the hole. In fact, the state that takes over Nagaoka's state at $\Phi_{0} / 2$ is a twisted spin structure, or a spin-density wave whose wavelength is as large as the sample size. The spiral structure is confirmed here from the spin-spin correlation for a $(\sqrt{26} \times \sqrt{26})$-site system displayed as an inset of Fig. 1 (a), where the spin polarization is seen to rotate gradually along the $y$ direction in which the AB flux exerts a twist.

Curiously, it is exactly this spiral state that is the first excited state from Nagaoka's at $\Phi=0$. Among the lowest-spin $(S=1 / 2)$ states, this (transfer-stabilized) spiral-spin state has a lower energy than the (exchange-stabilized) AF state until a level crossing occurs at a critical value of $U\left[\left(=167 t\right.\right.$, i.e., $J \equiv 4 t^{2} / U=0.024 t$ for a $(\sqrt{26} \times \sqrt{26})$-site system $\left.)\right]$ as $U$ is decreased.

The same occurs for a two-hole system, where the ground state starts off with the spiral spin state at $\Phi=0$ (for small enough $J$ ). [20] The state is characterized by a spin-spin correlation $S(\mathbf{Q})$ having four peaks at the wave number $\mathbf{Q}=\left( \pm 2 \pi / N_{x}, \pm 2 \pi / N_{y}\right)$ [inset of 
Fig. 2(b)]. The $S=0$ state changes into a two-peaked one continuously with $\Phi$.

Such twisted spin structures have in fact been analyzed to discuss the stability of Nagaoka's state. Douçot and Wen have introduced a twisted spin state, 21] where they assume that a hole hops on a frozen spin background. There, they have shown that a properly chosen spin texture can remove some of the frustration caused by the external flux. Although the Douçot-Wen function is an approximate trial one, which is not even an eigenstate of the total spin, the function, when cast into our Bloch form, does serve a heuristic purpose of identifying two kinds of Bloch momenta. We can first call $\mathbf{k}$, which is coupled to $\mathbf{x}_{\mathrm{h}}$ in Eq. (2), the charge Bloch wave number. The Douçot-Wen spiral configuration is frozen in the rest frame, but is now specified by the position of its loop $\mathbf{r}_{0}=\left(x_{0}, y_{0}\right)$ (where the spin wave function has a phase slip of $\pi$ ) in our frame moving with the hole. We can take a linear combination of spin configurations having different $\mathbf{r}_{0}$ with a spin Bloch wave number q. Let $\theta=2 \pi / N_{y}$ be the wave number of the spin twist in the Douçot-Wen function. The variational function then reads

$$
|\varphi\rangle=\sum_{\mathbf{x}_{\mathrm{h}}} \exp \left(\mathrm{ik} \cdot \mathbf{x}_{\mathrm{h}}\right) \frac{1}{N} \sum_{x_{0}, y_{0}} e^{i \mathbf{q} \cdot \mathbf{r}_{0}} \prod_{\substack{(x, y) \\
\neq \mathbf{x}_{\mathrm{h}}}} \otimes\left|\begin{array}{c}
\cos \left[\frac{\theta}{2}\left(y+y_{0}\right)\right] \\
\sin \left[\frac{\theta}{2}\left(y+y_{0}\right)\right]
\end{array}\right\rangle_{(x, y)}
$$

where $\left.\left.\right|_{\beta} ^{\alpha}\right\rangle_{(x, y)}$ is the spinor at site $(x, y)$ and we have again assumed that the order in the product $\prod_{(x, y)}$ is according to the rule described below Eq. (2) . If we choose $\mathbf{q}=(0, \theta / 2)$ for the spin Bloch wave number, the variational energy becomes

$$
\varepsilon=-2 t \cos k_{x}-2 t \cos \frac{\theta}{2} \cos \left(k_{y}+\frac{2 \pi \Phi}{N_{y} \Phi_{0}}-\frac{\theta}{2}\right),
$$

for $U=\infty$, and is minimized at the half-flux, where the difference between the AB phase $2 \pi \Phi / N_{y} \Phi_{0}$ and the spin phase $\theta / 2$ vanishes.

Thus we are led to a picture in which the extra phase $\theta / 2$ arising from the gauge potential (an internal frustration) from the spin configuration acts to cancel the enhancement in the kinetic energy caused by the external (flux-induced) frustration. There, the low-spin states becomes advantageous for the maximal (half-flux) frustration, since they have the maximum degrees of freedom in the spin space that enable them to absorb the frustration. 
We expect this should generally apply to strongly correlated systems with restricted motion of charges (i.e., excluded double occupancies), so that the variation of the energy, and thus the persistent current, for spinfull electrons should be smaller than those for (the same number of) spinless electrons.

To be more precise, the spin configuration is disturbed by the hole motion as stressed [22], and a factor neglected by the Douçot-Wen wave function is the quantum fluctuation. This is numerically seen in a reduction in the absolute value of the nearest-neighbor spin-spin correlation from that for the spiral configuration of classical $S=1 / 2$ spin in the inset of Fig. 11(a). However, the inseparability of the center-of-mass and internal coordinates and their interference does in general remain when the configurational subspace classified by the total orbital angular momentum and the spin are 'irreducible' (i.e., when any two states within the subspace are connected by a series of nonzero matrix elements of the transfer). Thus, in addition to the well-known enhancement in the effective mass of a hole, this gives rise to the spin-modulated persistent current in clean two-dimensional lattices, unlike two-dimensional continuum systems.

In other words, a current deviating from the free-electron behavior should be generic to any lattice systems, such as the $t-J$ model, that have a constrained electron motion due to strong interactions. For example, in the AF phase in the finite- $U$ Hubbard model or $t-J$ model in 2D, $E_{i}(\Phi)$ deviates from the free-electron result as shown by Poilblanc [23]. A difference there from the present situation is that the level crossing is shown to occur among the exchange-stabilized $S_{t o t}=0$ states (i.e., within the AF phase). There, we also observe a reduction in the deviation of the ground-state energy, which may again be explained from the flux-induced enhancement of the kinetic energy as relaxed by the gauge field arising from the spin wave function.

Obviously, another important question is the effect of disorder or the edge effect for open geometries to make the model approach to actual mesoscopic systems. As for the ferromagnetism, whether there is a finite ferromagnetic region around Nagaoka's limit is still an open question. A finite region for the partial polarization is proposed from a $t$ - 
$J$ study [24], but a possibility of an abrupt transition to an AF state is also suggested [20]. However, we can provide a way to relax the singular situation of Nagaoka's by going over to two-band Hubbard models, where the double-exchange mechanism induces a more stable ferromagnetism that we can show has close similarities with Nagaoka's [25]. Another prominent example of the stabilized ferromagnetism is the Hubbard model on flat-band systems 26]. There the flat band, which is fully polarized when half-filled, becomes metallic when doped, and it will be interesting to see how the persistent current behaves in such an itinerant magnet.

The authors would like to thank Professor Yshai Avishai for discussions. Numerical calculations were performed on HITAC S3800 in the Computer Center, University of Tokyo. This work was supported, in part, by a Grant-in-Aid from the Ministry of Education, Science and Culture, Japan. 


\section{REFERENCES}

[1] V. Ambegaokar and U. Eckern, Phys. Rev. Lett. 65, 381 (1990); 67, 3192 (1991).

[2] D. Loss, Phys. Rev. Lett. 69, 343 (1992).

[3] R.A. Smith and V. Ambegaokar, Europhys. Lett. 20, 161 (1992).

[4] M. Abraham and R. Berkovits, Phys. Rev. Lett. 70, 1509 (1993).

[5] A. Müller-Groeling et al, Europhys. Lett. 22, 193 (1993); A. Müller-Groeling and H.A. Weidenmüller, Phys. Rev. B 49, 4752 (1994).

[6] R. Berkovits, Phys. Rev. B 48, 14381 (1993).

[7] G. Bouzerar et al, Phys. Rev. B 49, 8258 (1994).

[8] H. Kato and D. Yoshioka, Phys. Rev. B 50, 4943 (1994); D. Yoshioka and H. Kato, (unpublishd).

[9] R. Berkovits and Y. Avishai, Solid. State Commun. 93, 301 (1995).

[10] B.S. Shastry and B. Sutherland, Phys. Rev. Lett. 65, 243 (1990).

[11] D. Loss and P. Goldbart, Phys. Rev. B 43, 13762 (1991).

[12] F.V. Kusmartsev, Phys. Lett. A 161, 433 (1992); F.V. Kusmartsev et al, Phys. Rev. B 49, 16234 (1994).

[13] S. Fujimoto and N. Kawakami, Phys. Rev. B 48, 17406 (1993).

[14] Y. Nagaoka, Phys. Rev. 147, 392 (1966).

[15] J.A. Riera and A.P. Young, Phys. Rev. B 40, 5285 (1989).

[16] N. Byers and C.N. Yang, Phys. Rev. Lett. 7, 46 (1961).

[17] A. Barbieri et al, Phys. Rev. B 41, 11679 (1990).

[18] K. Kusakabe and H. Aoki, J. Phys. Soc. Jpn. 61, 1165 (1992). 
[19] In one dimension, $\mathcal{T}_{a}$ reduces to a rotation on the ring for one hole, but the spin momentum, $2 n \pi /(N-1)(n$ : an integer), is incommensurate with $k$ resulting in an eigenenergy, $-2 t \cos \left(k+2 \pi \Phi / N \Phi_{0}-2 n \pi /(N-1)\right)$. Although we need the Bethe-ansatz solution for general $N_{\mathrm{e}}$ (Ref. [12]), $N_{\mathrm{e}}$-flux periodicity is derived for $N_{\mathrm{e}}=N-1$ already from the above expression.

[20] K. Kusakabe and H. Aoki, Phys. Rev. B 50, 12991 (1994).

[21] B. Douçot and X.G. Wen, Phys. Rev. B 40, 2719 (1989).

[22] As a consequence $\varepsilon$ cannot reach $-4 d t$ as seen from another reduction factor $\cos (\theta / 2)$ in Eq.(5).

[23] D. Poilblanc, Phys. Rev. B 44, 9562 (1991).

[24] W.O. Putikka et al, Phys. Rev. Lett 69, 2288 (1992).

[25] K. Kusakabe and H. Aoki, unpublished.

[26] K. Kusakabe and H. Aoki, Phys. Rev. Lett 72, 144 (1994) and references therein. 


\section{FIGURES}

FIG. 1. (a) AB flux $(\Phi)$ dependence of the lowest state for each of various total spins in a one-hole doped $(4 \times 4)$-site system with $U=\infty$, in which the ground state evolves from a ferromagnetic state at $\Phi=0$ down to a spiral-spin state at $\Phi=\Phi_{0} / 2$. (b) The same for a two-hole doped $(4 \times 4)$-site system. The insets depict the spin-spin correlation function $\langle S(\mathbf{0}) S(\mathbf{R})\rangle$ for the spiral-spin ground state with the half-flux in a one-hole doped $(\sqrt{26} \times \sqrt{26})$-site system [the arrow in (a)] and the spin structure factor $S(\mathbf{Q})$ for the ground state without flux in a two-hole doped $(\sqrt{20} \times \sqrt{20})$-site system [the arrow in (b)] at $U=\infty(J=0)$.

FIG. 2. The persistent current derived from the previous figure via the Byers-Yang theorem for the $(4 \times 4)$-site Hubbard model for one hole (a) or two holes (b) with $U=\infty$. 